\title{
Evaluation of Apical Microleakage of Endodontically Treated Teeth Sealed With Three Different Root Canal Sealers
}

\author{
Omar S. Rahawi \\ BDS, MSc (Lect.) \\ Manaf B. Ahmed \\ BDS, MSc (Assist Lect.) \\ Sabah A. Ismail \\ BDS, MSc (Assist Prof)
}

\author{
Department of Conservative Dentistry \\ College of Dentistry, University of Mosul \\ Department of Conservative Dentistry \\ College of Dentistry, University of Mosul \\ Department of Conservative Dentistry \\ College of Dentistry, University of Mosul
}

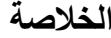

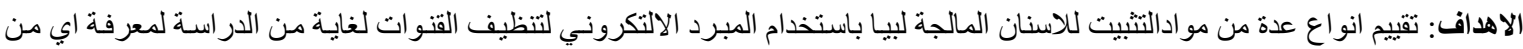

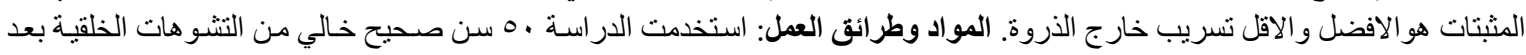

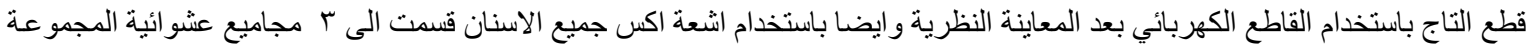

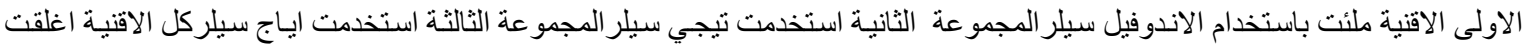

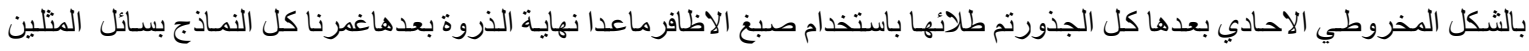

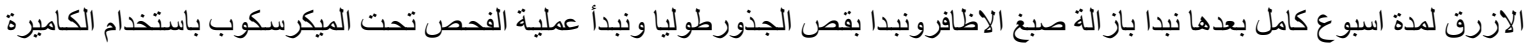

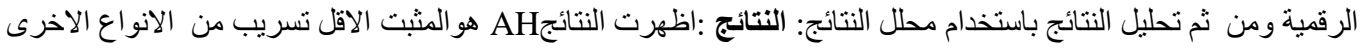

\begin{abstract}
Aims: Different materials are used during endodontically treating of teeth as root canal sealers. Thus, this study was done to evaluate the sealing ability of three different types of root canal sealers on the apical part of the tooth. Materials and Methods: 50 sound human teeth were instrumented by using a rotary ProTaper nickel-titanium files under crown down technique. The samples were divided randomly into 3 groups $(n=10)$, additionally two groups were used as +ve controls, first group usedEndofill sealer (PD product dentairesSA,Switzerland), and group two used Tagadseal sealer (technical general United kingdom), and the third group used AH plus sealer (Dentsply, Switzerland). All teeth were obturated using the single cone technique with the sealers, then the whole root surface was coated with two layers of aspecial type of nail varnish, except the apical $1 \mathrm{~mm}$, then all teeth were placed into $2 \%$ methylene blue dye liquid for one week at $37^{\circ} \mathrm{C}$. After that they were removed from the dye , the nail varnish was removed. The positive controls $(\mathrm{n}=10)$ were left unfilled and coated as described earlier. Then the tooth was splited longitudinally and each half examined under a stereomicroscope with magnification: $10 \mathrm{X}$. A computer software was ued taken by a digital camera. Analyzing of data was done by One-Way Anova and Duncan's Test analysis among the means of microleakage of the three types of sealers. All tests were computed at 5\% significance level.Results: showed statistically significant difference among groups. Group 3 (AH plus) showed abtter seal among ( $\mathrm{Tg}$ and Endofill) groups while Tg and Endofill showed no significant difference between both. Conclusions: Under the conditions of this study, the AH plus sealer provided a better sealing ability than Endofill and Tagadseal sealers, but long term in vivo study is important to prove it.
\end{abstract}

Key words Apical sealing, root canal sealer, AH plus sealer.

Rahawi OS, Ahmed MB, Ismail SA: Evaluation of Apical Microleakage of Endodontically Treated Teeth Sealed With Three Different Root Canal Sealers. Al-Rafidain Dent J. 2019;19(1):11-19.

Received: 15/7/2019 Sent to Referees: 20/9/201 Accepted for Publication: 2/12/2019 


\section{INTRODICTION}

The main objective of endodontic treatment is to obtain the full elimination of microorganisms from the canal of the root and to obtainan apical, lateral, and coronal seal to prevent colonization by the microorganisms. Failure to seal the apical part of the root has accounted for as many as $60 \%$ of endodontic treatment failures $^{(1)(2)}$.

Sealing of the root canal space is an important point for the success or failure of endodontic therapy, as it is related to the result of root canal treatment. It is an important point in enhancingperiapical healing and preventing disease progressing. Obturating materials as guttapercha has been considered the main standard for endodontic treatment as it has the least toxic and tissue irritating endodontic material available. However, guttaPercha cannot provide a lateral seal in the wall and for this reason a sealing agent is required. ${ }^{(3)} \mathrm{A}$ sealer for endodontic teeth require a permanent seal of the obturating material because it adheres the gutta-percha to the canal walls of the roots and fills all the irregularities and holes between gutta-percha cones and between the space of the root canal and fillings ${ }^{(4)}$.

The physical characteristics that are needed to be obtained from endodontic sealer arecapability to seal with the root canal space and gutta-percha with the body fluids and its stable after setting. For those reasons, more than one sealer will be developed to obtain the requirements of perfect sealing ${ }^{(1)}$. Various studies have been done on the sealing capability of many sealers and many levels of residual moisture in the root canal space have been shown to change the sealing characteristics of conventional and resin based sealers ${ }^{(5)}$. Therefore, this study was aimed to compare the apicalleakage of three commonly used root canal sealers cements.

\section{MATERIALS AND METHODS}

This study included 50 extracted monorooted with mono-canalled teeht. This features included mono canal with the curvature in the apical $\leq 20$ degree (Schneider method), apical constriction $\leq \mathrm{K}$-file \#20,complete and clean apex (under microscopic), and root canal opened. Exclusion feature were: root surface carious, closed canals and internal/external root resorption (clinically and $\mathrm{x}$-ray radiographs) Samples were washed and purified with a tooth micro brush under steamywater and kept in normal saline then a crown of the teeth were cut at the CEJ with a diamond bur disks under a water coolant to facilitate canal instrumentation.

Then, when cutting the crown the canal were opened and full length of the canals was obtained by inserting \#15 K-file insidethe canal until it was seen at the end of the foramen, and then subtracting $1 \mathrm{~mm}$ from the length ${ }^{(6)}$.

The root canals were prepared using a rotary ProTaper nickel-titanium files with 
crown-down method at $250 \mathrm{rpm}$ starting with Sx to S2 and F1 to F3 used the entire working length. Then canals were irrigated with $10 \mathrm{~mL}$ of a prepared solution of $5.25 \%$ sodium hypochlorite between each file. After instrumentation completion, a wash with 10 $\mathrm{mL}$ of $17 \%$ EDTA for one minute was used to clean the smear layer, then washing by $5.25 \%$ $\mathrm{NaOCl}$ then by distilled water. The canals were then dried by using sterile paper points.

After that samples were divided into 3 experimental groups $(n=10)$, Additionally two groups were used for positive and negative controls. The endodontic sealers were prepared according to instructions of the manufactures.. Three sealers were used in this study, the first group usedEndofill as a root canal sealer with dexamethasone ( PD product dentairesSA, Switzerland), and group two used Tagadseal resin based root canal sealer (technical general United kingdom), and the third group used $\mathrm{AH}$ plus root canal sealer (Dentsply, Switzerland).

All teeth were obturated using the single cone technique, with the using F3 protaper guttapercha dipped in the three sealers, excess gutta-percha was cleaned and condensed with a plugger $1 \mathrm{~mm}$ under the canal opening. The root canal opening was sealed with composite resin for better seal coronaly, then the whole root surface coated with twice, a special nail varnish away $1 \mathrm{~mm}$ from the apex, the all teeth were then placed into $2 \%$ methylene blue dye liquid for seven days at $37^{\circ} \mathrm{C}$. The samples were removed from dye solution, then teeth washed with running water and dried. The varnish was removed with a scalpel.

\section{Positive and negative controls:}

The positive controls $(n=10)$ were left unfilled and coated as described earlier. The negative controls $(n=10)$ were filled and totally coated, including the apical foramina.

The Canals obturated with gutta-percha (without sealer) and un layered with varnish are used as positive controls ( ${ }_{-}{ }_{10}$ ); the same roots are then entirely layered with special nail varnish for negative controls $\left(n_{-} 10\right)$.

\section{Measurement of micro-leakage:}

The roots of teeth were grooved longitudinally on each sides, using a diamond disc and handpiece (NSK, Japan) under cooling with distilled water. After positioning the location for splinting bucally lingually Divided along longitudinal axis near to the center of the canal and then teeth were split, and then each half examined with a stereomicroscope. To measure dye entrance, a stereomicroscope with magnification: $10 \mathrm{X}$ was used. The degree of leakage was measured from the end of the root to the highest amount of dye entrance in micrometers using computer software on the image captured by a digital camera as seen in Figures $(1,2)^{(7)}$. 


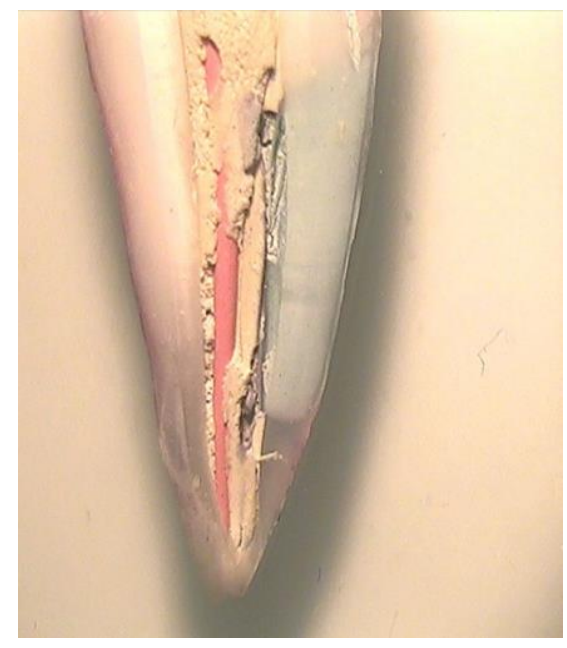

(1)

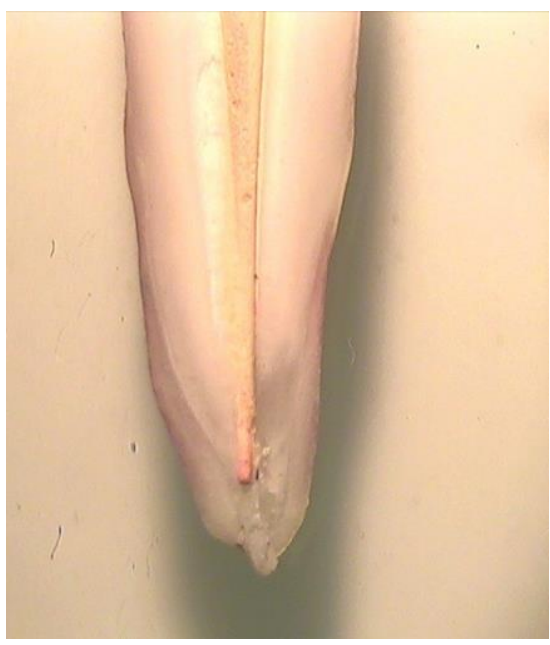

(2)

Figures (1,2): Pictures of teeth after splitting by digital camera of stereomicroscope.

\section{RESULTS}

The results of our study showed group 3 (AH Plus) to have the lowest mean value of dye pentration, whereas the highest mean value of dye penetration appeared in group 1 (Endofill). As in Figures $(3,4,5)$.

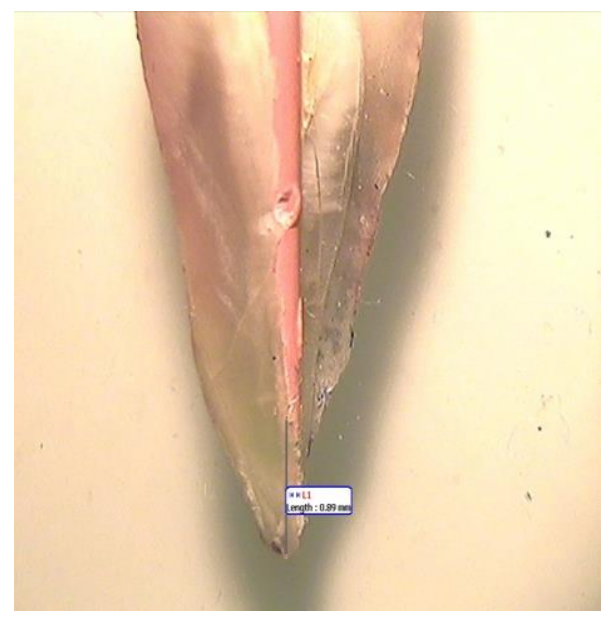

Figure (3): show degree of leakage of dye with $\mathrm{AH}$ plus sealer. 


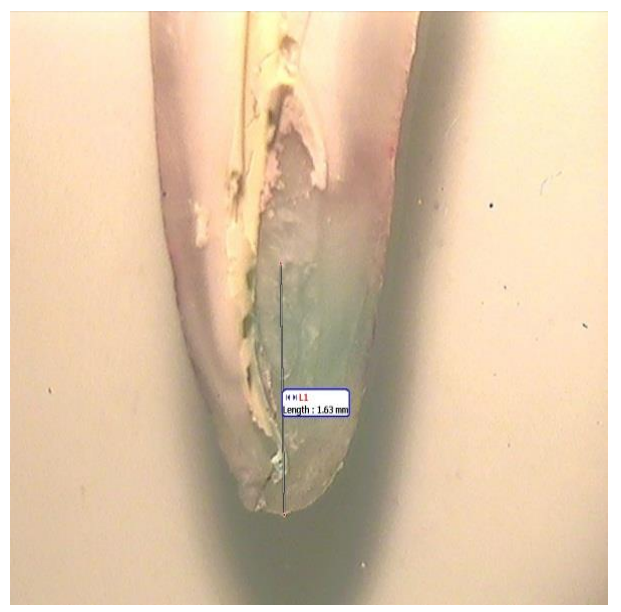

Figure (4): show degree of leakage of dye with Tagadseal sealer.

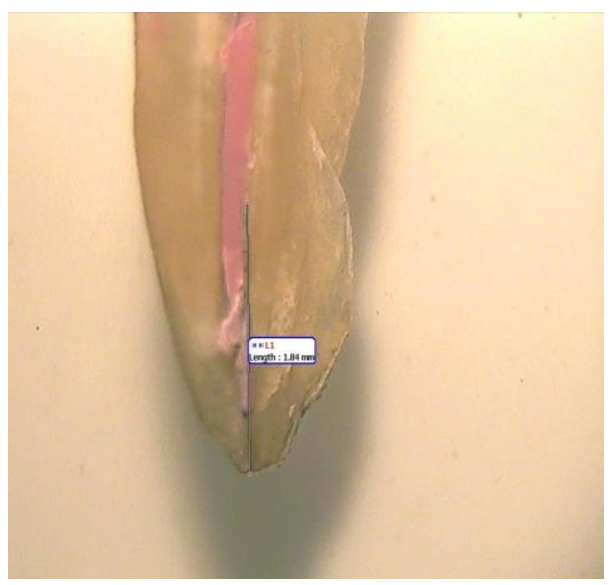

Figure (5): show degree of leakage of Endofill sealer.

Mean and standard deviation for the degree in groups appeared mostly at the junction of the of dye penetration for the study groups were endodontic sealer and root canal space in more measured as shown in Table (1), dye leakage of the samples.

Table (1): Descriptive analysis of the means of apical micro leakage of the three types of sealer

\begin{tabular}{llll}
\hline Type of Sealer & N & mean & SD \\
\hline Gp(3) AS & 10 & 0.20 & 0.40 \\
Gp (2) TS & 10 & 0.79 & 0.73 \\
Gp (1) ES & 10 & 1.22 & 0.30 \\
\hline
\end{tabular}

AS (AH Plus Sealer), TS (Tagadseal Sealer), ES (Endofill sealer). 
All data obtained evaluating analysis groups. Group 3 (AH plus) show less dye using parametric test One Way ANOVA and penetration among ( $\mathrm{Tg}$ and Endofill) groups Duncan's multiple range analysis test (Table while $\mathrm{Tg}$ and Endofill show no significant 2.3) respectively. One -Way Anova test show difference between them as shown in Table (3). statistically significant difference among

Table (2): One-Way ANOVA analysis among the means of microleakage of the three types of

\begin{tabular}{llllll} 
& \multicolumn{5}{c}{ sealers. } \\
& $\begin{array}{l}\text { Sum of } \\
\text { Squares }\end{array}$ & Df & Mean Square & F & Sig. \\
\hline Between & 5.245 & 2 & 2.622 & 10.013 & $.001^{*}$ \\
Groups within & 7.071 & 27 & .262 & & \\
Groups & 12.315 & 29 & & & \\
Total & & & & & \\
\hline
\end{tabular}

Df: degree of difference, F: F value at $\mathrm{P} \leq 0.05$, Sig: significantly*: Significantly highly.

Table (3): Duncan multiple range analysis test

\begin{tabular}{cccc}
\hline Groups (type of sealer) & $\mathrm{N}$ & Mean* $*$ SD & Duncan's groups** \\
\hline Gp (3) AS & 10 & $0.20 \pm 0.40$ & $\mathrm{~A}$ \\
Gp (2) TS & 10 & $0.79 \pm 0.73$ & $\mathrm{~B}$ \\
Gp (1) ES & 10 & $1.22 \pm 0.30$ & $\mathrm{~B}$ \\
\hline
\end{tabular}

AS (AH Plus Sealer), TS (Tagadseal Sealer), ES (Endofill sealer (*Mean in millimeter measurement $* *$ Different letter mean significant difference at $\mathrm{p} \leq 0.05$

\section{DISCUSSION}

An endodontic sealer is used to close the space between filling materialand root canal making a mass that can obtain a full seal. The apex of the root considered the most complex part of the root,becuse it has a large variation of anatomy like as ramifications, small canal, or lateral canals that make it increase the susceptibly of microorganism to leak ${ }^{(8)}$.
The goals of this study were evaluate the the apical adheringcapability of Endofill sealer, Tagadseal resin sealer and $\mathrm{AH}$ plus sealer. Sealing capability of the canal space of the root was measured by showing the line of coloring dye penetration on apex of the root.

The sealing efficiency of the endodontic sealer is observed by the adhering between the sealer and dentinal wall, and also by the physical 
characteristics of the endodontic sealer. Incomplete eradication of smear layer will affect the connection between dentin and sealer ${ }^{(9)}$.

The AH plus sealer showing best sealing ability. This was related to the characteristics of the AH plus sealer. The best characteristics of AH plusin related to Endofill and Tagadseal sealers, and high bonding feature of the sealer that showed in a high seal to dentin explains the high bondingcapability of the apical portion with that sealer. Whereas shrinkage after setting was shown with the other sealers, $\mathrm{AH}$ plus sealer was showing up to $1.0 \%$ expansion after settingsuggesting more adaptation and leastdye penetration at the space between tooth and filling. The $\mathrm{AH}$ plus sealer have low solubility can also enhance its sealing ability.

The results of this study agreed with those studies ${ }^{(10)},{ }^{(11)},{ }^{(12)},{ }^{(13)},{ }^{(14)}$.

Endodontic treatment of the root canal space with filling-material that has biocompatibility and lesser amount of shrinkage is very important for a perfect endodontic treatment. After that a full bond of the root canal space is almost impossible with materials and methods using a combination of gutta-percha and endodontic sealer, the endodontic sealer have the ability of making adherence between the filling material and the dentinal wall, that preventing leakage ${ }^{(15)}$.
3D sealing of the root canal space(sealing from al side ) was regarded the main goals of the root canal therapy and is important for avoiding re-infection of the tooth and for enhancing the health of the periradicular tissues, that obtained the successful of root canal therapy ${ }^{(16)}$.

More than one type of root canal sealers can be used to achieve this benefit and the evaluation of the apical bonding ability of the endodontic sealers is important. It should be observed that the sealing in the coronal part is of equal importance to the apical seal of the root canal for the success root canal treatment $^{(17)}$.

\section{CONCLUSIONS}

Many techniques can be used to evaluate the sealing ability of root canal materials. The evaluation of the linear dye leakage is the best technique used to shown the leakage of root canal fillings after splitting the $\operatorname{root}^{(18)}$.

\section{REFERENCES}

1. Bina A., Endang S. And Munyati U. Comparison of apical leakage in root canal obturation using bioceramic and Polydimethylsiloxane sealer (In vitro). Open journal of stomatology. 2018; 8:24-34.

2. Maham M., Farhan R. And MuneebA. Evaluation of Microleakage in Single-Rooted Teeth Obturated with Thermoplasticized GuttaPercha Using Various Endodontic Sealers: An 
In-Vitro Study. Journal of the College of Physicians and Surgeons Pakistan. 2018; 28 : 339-343.

3. Weizhang, zhili, and Bing pay. Assesment of new root canal sealer apical sealing ability.Oral radiolendol. 2009; 107:6-12.

4. Hadi M., Shahriar S., Maryam J., Mohammad F. , Hamid R.,Mokhtari Z. And Saeed R. Evaluation of Apical Leakage in Root Canals Obturated with Three Different Sealers in Presence or Absence of Smear Layer. Iranian Endodontic Journal. 2015; 10:131-134.

5. . Rupali B., . Sarvesha B. And . Vishal M. Evaluation of apical micro-leakage of Ah plus, MTA fillapex and ZOE sealers inthe presence and absence of moisture in root canals. International journal of current research. 2016; 8, Issue, 10:39883-39886.

6. Widcha A., Prapaporn Y., Kanittha K. And Noppakun V. Comparison of the Apical Sealing Ability of Calcium Silicate-Based Sealer and Resin-Based Sealer Using the Fluid Filtration Technique.Med PrincPract. 2016; 25:561-565.

7. Dr. Sundus H. The Sealing Ability of Guttaflow2 Sealer in Comparison to AH Plus Sealer Using Dye Penetration Method. Journal of Al Rafidain University College. 2015; 36: 337-347.

8. Chaudhari A., Asthana G., ParmarG., Vadher R. And Kaur M. Significant of Apical Third: A
Review. Scholars Journal of Applied Medical Sciences. 2014; 2:1613-1617.

9. Sonu K., Girish T., Ponnappa K.,Kishan K. And Thameem P. Comparative Evaluation of Dentinal Penetration of Three Different Endodontic Sealers with and without Smear Layer Removal (Scanning Electron Microscopic Study). Saudi Endodontic Journal. 2016; 6:16-20.

10. De Almeida W., Leonardo M., Tanomaru F., Silva L. Evaluation of apical sealing of three endodontic sealers. IntEndod. 2000; 33:25-7.

11. Joseph R. And Singh S. Evaluation of apical sealing ability of four different sealers using centrifuging dye penetration method: an in vitro study. J Contemp Dent Pract. 2012; 13:830-33.

12. Setia P., Sikri V., Sroa R. And Sidhu B. Apical Sealing Ability of Two Novel Root Canal Sealers: An ex-vivo Study. Journal of the International Clinical Dental Research Organization. 2013; 5:9-13.

13. Pawar S.,Pujar M. And Makandar S. Evaluation of the Apical Sealing Ability of Bioceramic Sealer, AH Plus \& Epiphany: An in vitro Study. Journal of Conservative Dentistry. 2014; 17:579-582.

14. Akcay M., Arslan H., Durmus N., Mese M. And Capar, I.D. Dentinal Tubule Penetration of AH Plus, iroot SP, MTA Fillapex, and 
GuttaflowBioseal Root Canal Sealers after

Different Final Irrigation Procedures: A Confocal Microscopic Study. Lasers in Surgery and Medicine. 2016; 48:70-76.

15. MokhtariH,Janani M. And Reyhani M. Evaluation of apical leakage in root canal obtured with three different sealer in presence and absence of smear layer. Iran Endo.J. 2015; 10:2-10.

16. RupaliB,Sarvesha $B$ and Vishal $M$. Evaluation of apical micro-leakage of AH plus ,MTA fillapex and ZOE sealer in the presence and absence of moisture in root canas. International Journal of Current Res. 2016; 8:10-15.
17. R.Vinodkumor and CS shruth. Evaluation of sealing ability of resin cement used as root canal study. Journal of Conservative Dentistry. 2012; 15:3-10.

18. Elmakki F.,Neamat H. And Ibrahim Y. Comparative study of micoleakage of resilon epiphany and guttpercha AH Plus obtrturating system.Iranian Endontic Journal. 2013; 7:1020.

19. George T.,Antonia I. And Burna M. Evaluation of apical leakage of white MTA associated with two different velicles. Journal health Sci.inst. 2010; 28:2-14. 